\title{
New constructive solutions for building of transport construction facilities
}

\author{
Vladimir Babayev ${ }^{1}$, and Valeriy Shmukler ${ }^{1 *}$ \\ ${ }^{1}$ O.M.Beketov National University of Urban Economy in Kharkiv, 61002, Kharkiv, Marshal \\ Bazhanov str., 17, Ukraine
}

\begin{abstract}
New structural systems for civil and transport engineering were examined. The basis for the formation of the proposed reinforced concrete structures is the ideology of reducing its dead weight, with a given bearing capacity, the realization of which is accomplished by burial during concreting large-sized liners of a given shape and manufactured from lightweight, inexpensive composite materials. The process of erecting these systems is presented in two forms: for flat structures - using selftightening concrete, and for curvilinear ones - by using shotcrete technologies. The second direction is presented by steel-reinforced concrete structures. These structural systems were created on the basis of innovative component and methods of rationalization of parameters. The basis of the above methods is a compiler which includes the finite element method, adaptive evolution method and special iterative procedures. Experimental verification of structural solutions and formation procedures for suggested systems was performed. Comparison between theoretical and experimental data is given. Suggested systems were implemented in a number of building companies.
\end{abstract}

\section{Introduction}

Search for new advanced constructive solutions and efficient technologies for their implementation is predetermined with the complex of requirements for state-of-the-art construction projects combined with limited resources. At the same time, the dual "designtechnology" task means constructing systems that provide a wide variability of special planning solutions, contain necessary properties of environmental positivity, resistance to environmental variability, and economic expediency.

Nevertheless, the processes occurring in the world around us (climate transformations, seismic activity, abnormities of artificial origin, etc.) and the population increase bring to the foreground the issues which have hitherto had rather local, than global solution.

They include, first of all, the need to allocate better lands for agricultural and forestry needs, reconstruction of existing and introduction of new sources of energy, saving of drinking resources and, consequently, creation of "intelligent" buildings and structures [1]. This is the basis for the integrated design of individual elements and structural systems as a whole in a logical combination with engineering support.

*Corresponding author: konstruktsii@gmail.com 
The above said is regarded as a reason for creation and implementation of new constructive systems, the main features of which are discussed below.

\subsection{General}

The need to improve the covering and flooring design, as well as pedestrian bridges, predetermines the search for fast-erecting systems built from large factory-assembled elements. The above is consistent with R. Descartes' concept involving the thesis that any saving is ultimately a time saving.

Steel-reinforced concrete constructions are known to have certain positive prospects here [2]. However, heavy weight of the reinforced concrete part worsens the amplitude frequency response of the structure, thus contributing to increase in dynamic forces (including seismic) and/or occurrence of resonance modes. In addition, the criterion worsens characterizing the ratio of the share of the resource spent for perception of its own weight and those spent for perception of the payload.

The above was the reason for creation of a system that combines efficient design and technological solutions.

\subsection{Design and technological process features}

The suggested flooring construction is a spatial flat parallel slab-and-rod system (structure) made of two materials: reinforced concrete and steel $[3,4,5,6]$. The specific feature of the reinforced concrete slab is a profiled steel sheet used as external reinforcement, which is also a non-removable formwork. The sheet is fixed in the plane of the upper boom of the structure by special shear connections and either self-tapping screws or electric plugs. Another fixing element here is cold-drawn wire mesh reinforcement. Lightweight concrete is usually used. Using prefabricated reinforced concrete slabs of the "per cell" size is possible.

The specific feature of the metal structure is its manufacture of flat elements of the "span" size, which simplifies significantly the design of the unit and the on-site assembly, minimizing the time and labour intensity of the assembly. The feasibility of this structure is limited to the span of $60.0 \mathrm{~m}$. The essence of the design solution of this dual aggregate is as follows: formation of a structure consisting of metal modular elements, upper and lower booms, and reinforced concrete monolithic or prefabricated slabs (Fig. 1, 2, 3).

Each modular element is a truss with parallel chord. Truss booms are made from single angles rotated at $45^{\circ}$ to the vertical axis. Lattice rods generally have closed cross-section and are attached to metal gusset plates. During the on-site assembly, modular elements are installed rotated at an angle of $45^{\circ}$ to the horizon and bolted together. If necessary, longitudinal girders made of metal profiles located perpendicularly to the modular elements can be arranged above and below the resulting fold system. In this case, the upper girders together with the booms of the modular elements form closed sockets, into which the reinforced concrete prefabricated slabs or profiled sheet and reinforcing mesh are laid. In the latter case, concrete is poured on the mesh reinforcement. The above girders in combination with the angle elements and mesh reinforcement are shear connections that ensure the joint performance of the composite (monolithic) reinforced concrete disc with the rods of the spatial metal structure. The lower booms unite the modular elements along the lower plane of the structure. Additional positive feature of the suggested solution is the fact that the angle element (shear coupling) is manufactured using the non-wastes technology for triangle plates (Fig. 3). The principal unit of the modular element is shown in Figure 1. In particular, a nonfigured truss can be formed placing a gusset plate between the feathers of the flange angle. This significantly improves the architectural expressiveness of the system being created. In this case, the nodal element is a "table" consisting of a rectangular slab and three triangular 
bearing plates welded into the boom angle from inside. Furthermore, a positive fact here is a complete two-sided closure of the tubular cross-section of the brace, which minimizes significantly the corrosion processes in these elements.

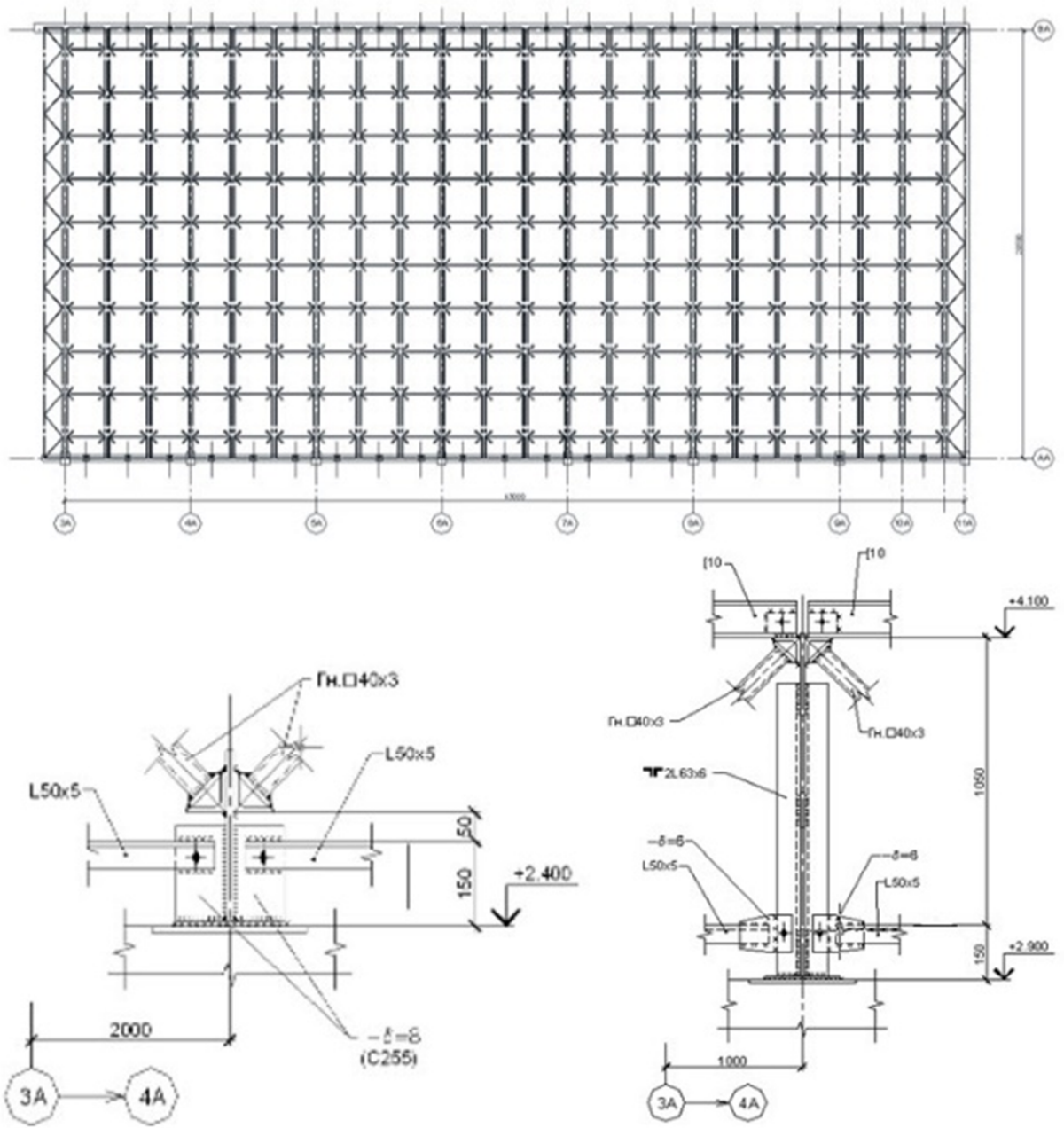

Fig. 1. Flooring arrangement. Flooring diagram. Cross-sections.

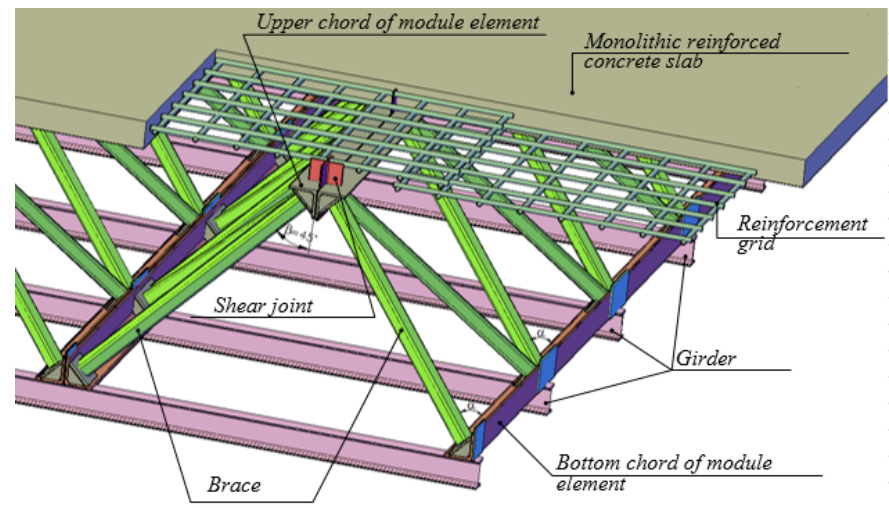

Fig. 2. Suggested flooring design. 
The reliability of the suggested design is provided by the same (standard) solution of the connection joints of both upper and lower chords, reduction of welded joints performed on the site, absence of welded joints between the slabs located in the plane of the upper boom and the lattice rods. Simplification, accuracy and speed of assembly are achieved due to rotation of the booms of modular elements by $45^{\circ}$ to their vertical axis, which, consequently, ensures a tight fit of the upper and lower booms of modular elements to each other during assembly. Thus, the considered design is simultaneously a conductor for its own assembly. Rational use of the applied materials is ensured by the appropriate location of the reinforced concrete (slab) and the metal (lattice) parts of the structure.

This is due to the fact that the monolithic reinforced concrete slab or prefabricated slabs are located in the upper chord plane and are completely in the compressed zone of the structure (Fig. 2, 3).

a)

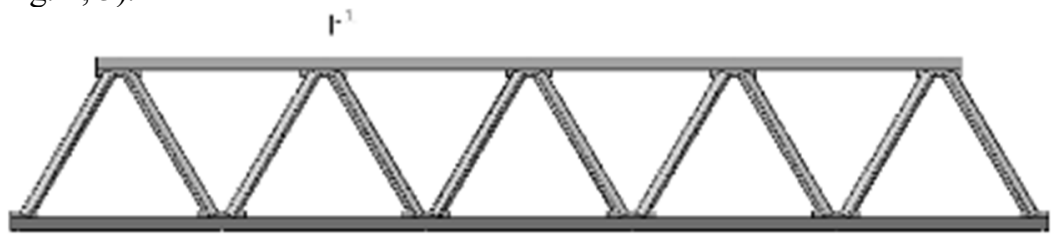

b)

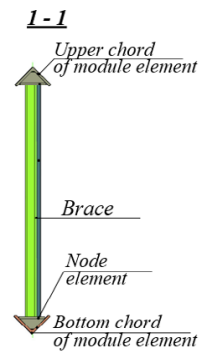

c)

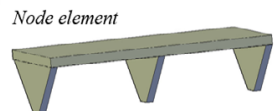

d)

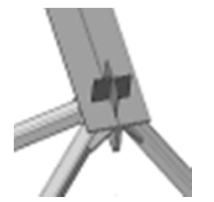

e)

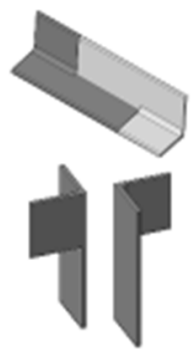

Fig. 3. Properties of the flooring construction: a) modular element; b) cross-section 1-1; c) beating "table"; d) assembly of shear connection; e,f) construction of shear connection.

The assembly of the metal-concrete spatial flooring is reduced to the direct connection of modular elements (trusses). They are connected first using bolts, and then welding, in the limited number of points.

Liners from a light, inexpensive material (expanded polystyrene, mineral wool, etc.) are laid inside the reinforced concrete slab when concreting to reduce its dead weight. Such a solution allows reducing the weight of this element to $50 \%$, without significant complication of the technological process [7].

\subsection{Simulation of a stress-strain state and rationalization of parameters}

The theoretical basic of algorithms and procedures for determining rational parameters of the considered construction is the suggested methodology for constructing iterative computational sequences [8].

It is based on a compilation that synthesizes the finite element method and the adaptive evolution method $[9,10,11]$. Combination of the finite element method and the method of regulation by the system parameters, especially in the form of the adaptive evolution method, enables to construct a direct determination procedure for physical and geometric characteristics of the structures. The developed algorithm assumes the determination of internal system parameters as a result of the sequential construction of a system with equal energy strengths. The optimized, equable system in known to minimize the construction material consumption [10]. 
The above said defines the concept of a rational design as a system for which in all elements the condition $e=$ consl ( $e$ - deformation energy density) is fulfilled. Also, for the least material intensive systems $e=e_{u}\left(e_{u}\right.$ - ultimate deformation energy density) [9].

The above compiler predefines iterative clarification of geometric and/or physical and mechanical system parameters in accordance with the conditions $e \rightarrow e_{n}\left(e_{n}\right.$-rated value of deformation energy density). In this case, the finite element method is used at each step (of the iteration process), while the evolution adaptation means the possibility of accounting the values of calculated parameters of any previous steps at the $i$ th step (hereditarity).

With the dependence $\langle\sigma-\varepsilon\rangle(\sigma-$ stress, $\varepsilon-$ strain $)$ and ignoring the load alleviation and elastic recovery, we can determine the strain energy density using the formula:

$$
e=\int_{0}^{\varepsilon^{*}} \sigma(\varepsilon) d \varepsilon \leq e_{n},
$$

where $\varepsilon^{*}$ - current strain value.

In turn, the unknown internal parameters affect the configuration and volume, and are determined based on the hypothetical expression:

$$
h^{i+1}=h^{i}\left(e^{i} e_{n}^{-1}\right)^{\gamma}
$$

where $h$ - corresponding internal parameter, for example, the cross-sectional height of the slab or the cross-sectional area of the rods;

$i$ - number of the internal (current) iteration;

$\gamma$-adaptive evolution parameter, while, $\gamma €[0,1]$ and is determined based on the simulation experiment $[10,13]$.

The general procedure for solving the formulated problem now is written as:

$$
\begin{gathered}
A_{i} \in\left\lfloor A_{i}^{\min } ; A_{i}^{\max }\right] ; \delta_{r}^{m, n} \in\left[\delta_{\min } ; \delta_{\max }\right] \Rightarrow\left\{q^{m, n}\right\}= \\
{\left[R^{m, n}\right]^{-1}\left\{P^{m, n}\right\} \Rightarrow e_{r}^{m, n} \Rightarrow \Rightarrow \delta_{r}^{m, n+1}=\left(e_{r}^{m, n} e_{n}^{-1}\right)^{\gamma} \cdot \delta_{r}^{m, n} ;} \\
A_{r}^{m, n+1}=\left(e_{r}^{m, n} \cdot e_{n}^{-1}\right) \cdot A_{r}^{m, n}, \\
U^{L}=\inf _{\alpha} U(\alpha) \\
\mathrm{m}=1,2, \ldots \mathrm{L} ; \mathrm{n}=1,2, \ldots \mathrm{S}
\end{gathered}
$$

where: $R^{m, n}$ - system stiffness matrix; $\left\{q^{m, n}\right\}$ - displacement vector; $\left\{P^{m, n}\right\}$ - outside force vector; $A_{i}$ - cross-sectional area of the rod (internal parameter); $\delta_{r}$ - characteristic dimension of the $\mathrm{r}$-th finite element (internal parameter); $U_{r}^{m, n}$ - potential strain energy of $\mathrm{r}$-th finite element; $V_{r}$ - volume of $\mathrm{r}$-th finite element; $A_{i}^{\min } ; A_{i}^{\max } ; h_{\min } ; h_{\max }$ - known invariables; $e_{r}^{m, n}$ - potential deformation energy density in the r-th finite element; $S$ - number of internal cycle iterations; determined in the counting order until the inequation is fulfilled:

$$
\left|V^{n+1}-V^{n}\right| / \min \left\{V^{n+1} ; V^{n}\right\} \leq \mu,
$$


$L$ - number of external cycle iterations; $\mu$ - known invariable; $V$ - total adduced element volume.

To determine external parameters of the construction $\alpha_{i}(i=1,2,3 \ldots \mathrm{m})$, let us write the system of balance equations as the finite element method:

$$
R\left(\alpha_{i}\right) \cdot q=P .
$$

The system (5) contains $n$ unknown nodal displacements and $m$ unknown external system parameters, which are included in (5) in the non-linear manner. In [9], it is suggested to choose (at each iteration step) from the whole set of possible values of unknown parameters those at which potential energy of strain (PED) reaches the lower limit, i.e.

$$
U=\inf U^{k}, \mathrm{k}=1,2 \ldots
$$

where $U$ - potential energy of strain of the system, $k$ - variant number.

Let us accept inclination angles of the braces of the structure as external parameters and calculate the value of potential energy of strain of the construction $U=U(\alpha, \beta)(\alpha, \beta-$ inclination angles of the braces in the mutually perpendicular planes, external parameters). Let us write the system of nonlinear equations

$$
\left\{\begin{array}{l}
\frac{\partial U(\alpha, \beta)}{\partial \alpha}=0 \\
\frac{\partial U(\alpha, \beta)}{\partial \beta}=0
\end{array} .\right.
$$

Its solutions determine $\alpha$ and $\beta$ values, corresponding to inf $U$ and define minimum consumption of materials. The system of (7) equations is simplified for the considered construction, since the angle between the modular elements is known before and is $\beta=45^{\circ}$. This fact is predetermined with the flooring design. Thus, for the parameters included in (1.7), we have:

$$
\begin{gathered}
U=\sum_{i=1}^{k} \frac{N_{i}^{2} L_{i}}{2 E_{i} A_{i}}, \quad A_{i}=\left|N_{i}\right|[\sigma]_{i}^{-1}, \\
U=\sum_{i=1}^{k} \frac{1}{2 E_{i}}[\sigma]_{i}\left|N_{i}\right| L_{i}
\end{gathered}
$$

and (7) acquires the form:

$$
\frac{\partial U}{\partial \alpha}=U_{o} \frac{\partial \varphi(\alpha)}{\partial \alpha}=0, U=U_{0} \varphi(\alpha),
$$

where $N_{i}$ - stress in the $i$-th $\operatorname{rod} ; L_{i}-$ rod length; $[\sigma]_{i}$ - permissible stress; $E_{i}-$ stressstrain modulus of the 1 st genre of the rod material; $A_{i}$ - cross-sectional area of the rod; $k-$ number of rods in the system; $U_{0}$ - known invariable; $\varphi(\alpha)$-dimensionless function of the inclination angle of the brace $\alpha$.

Calculations for modular elements of different height demonstrate that the function $\varphi(\alpha)$ around the extreme point hardly deviated form the right line parallel to the axis of abscissas 
(Fig. 4). This fact provides for using discrete assortment without significant increase of consumption of materials. However, expressions (8), (9) defining minimum weight of the construction are valid only if its constant for all rods of the deformation modulus system of the $1^{\text {st }}$ genre and permissible stresses, i.e. $E=$ const, $[\sigma]=$ const. This fact is a consequence of connection linearity for statically determinable trusses between the minimum volume of the rod system and the minimum potential energy value:

$$
U=f V,
$$

$V$ - total volume of the rod material; $f=\frac{[\sigma]^{2}}{2 E}-$ known invariable.

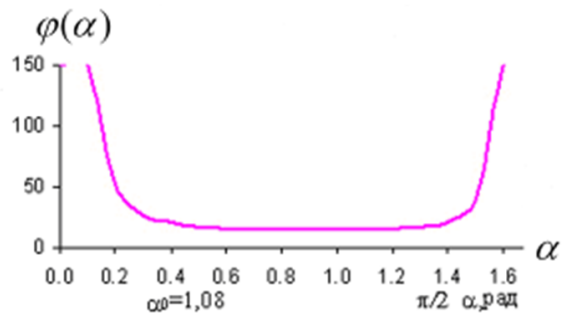

Fig. 4. Connection between the potential deformation energy of the system and inclination angles of the brace.

Thus, the given algorithm implements definition of the indicated inclination angle of the rods at the given height of the construction. The external parameter can be estimated using discrete search of the height.

The solution obtained with (3), in general, does not satisfy limitation for displacements, crack width, self-induced vibration frequencies, technology etc. This fact results in the necessity to increase cross-sections of the element with equal energy strengths.

In this connection, an additional task is formulated as follows:

$$
\begin{gathered}
\Delta V(\{\alpha\}) \rightarrow \inf , \\
\omega^{\min } \geq \omega_{a d m} ; a_{c r c}^{\max } \leq a_{a d m} ; f^{\max } \leq f_{a d m},
\end{gathered}
$$

where $\Delta \mathrm{V}-$ extra volume of the material; $\{\alpha\}-$ internal parameter vector; $\omega^{\max }, \omega_{c r c}^{\max }, f^{\max }$ - minimum (maximum) value of self-induced vibrations or crack width or deflection; $\omega_{\text {adm }}, a_{a d m}, f_{a d m}$ - known invariables, normally accepted from the national standards.

The problem (11) - (12) is solved by search for a new, in relation to the found one, equal-energy state of the system, at which $e<e_{n} \wedge e=$ const . Topology of the system with equal energy strengths $\left(e=e_{n}\right)$ is accepted as first approximation.

Further, fulfillment of limitations (12) is checked for this solution. If they are fulfilled, this solution can be consider final. In general, first approximation will not be a solution (11)-(12). Then a notion of conditional ultimate strain energy density $e_{n m}$ is introduced ( $m-$ number of inequations in (12)).

Then the procedure is constructed as the Kiffer-Johnson's algorithm to reach the boundary of the region of feasibility [13]. As a result of the operation of the considered iterative algorithm, the geometry and/or physical and mechanical characteristics of the structure are determined. 


\subsection{Experimental testing}

Experimental verification of the elaborated system and methods for its calculation was carried out. The non-destructive tests were conducted at an ambient temperature of $30^{\circ} \mathrm{C}$ and humidity of about $60 \%$. A research object was a fragment of the superstructure of a pedestrian bridge with a length of $7.0 \mathrm{~m}$, a width of $2.0 \mathrm{~m}$, and a construction height of $0.5 \mathrm{~m}$ was adopted (Fig. 5). The hydraulic testing (water loading) method was adopted as a loading method [13]. Therefore, a tank was arranged on the upper surface of the slab, allowing short-term and long-term loading on the entire structure, its half and quarter. Loading was performed in steps from $1 \mathrm{kPa}$ to $8 \mathrm{kPa}$ in increments of $1 \mathrm{kPa}$, followed by unloading (low-cycle loading). The instruments included 78 resistance sensors, 13 deflection meters, 4 dial indicators, 10 displacement sensors sensors controlled with SIIT-3 information and measuring system and a PC with the appropriate software installed (Fig. 7).
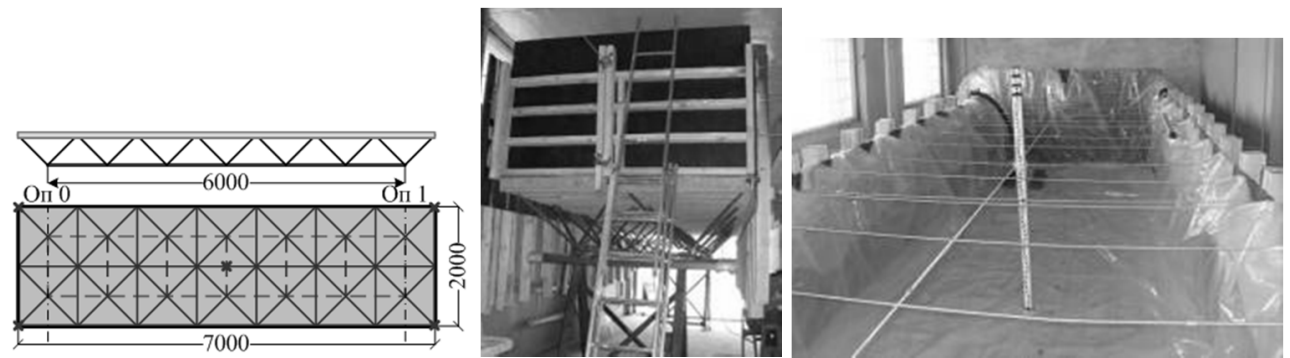

Fig. 5. Loading of the entire construction.

Deformations and displacements were measured in the horizontal and vertical direction of the construction, as well as on individual elements (such as slab, booms, braces). In addition, a possible shift of the reinforced concrete slab relative to the metal structure was to be determined. The results of the measurements are illustrated by the diagrams in Figures 5,6 and 8 .
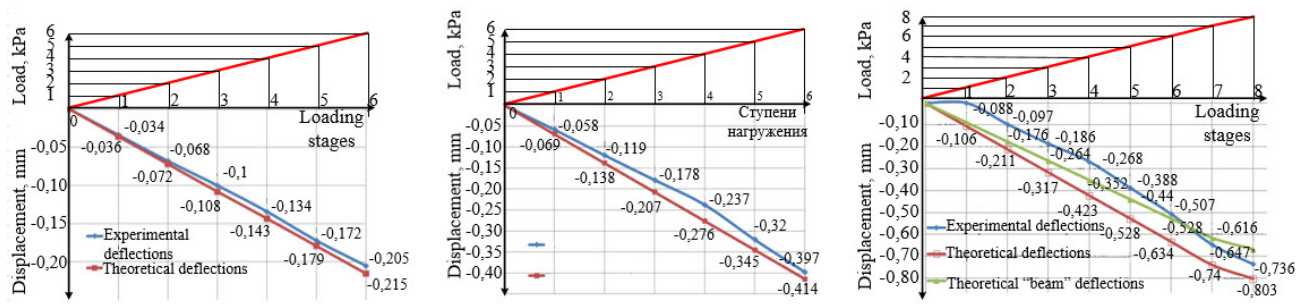

Fig. 6. Maximum displacements in the middle of the span at short-term and long-term loading.
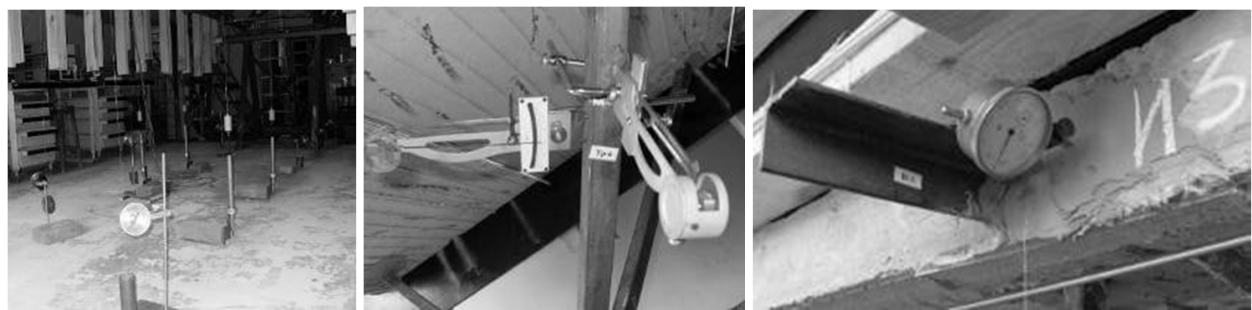

Fig. 7. Available instruments. 


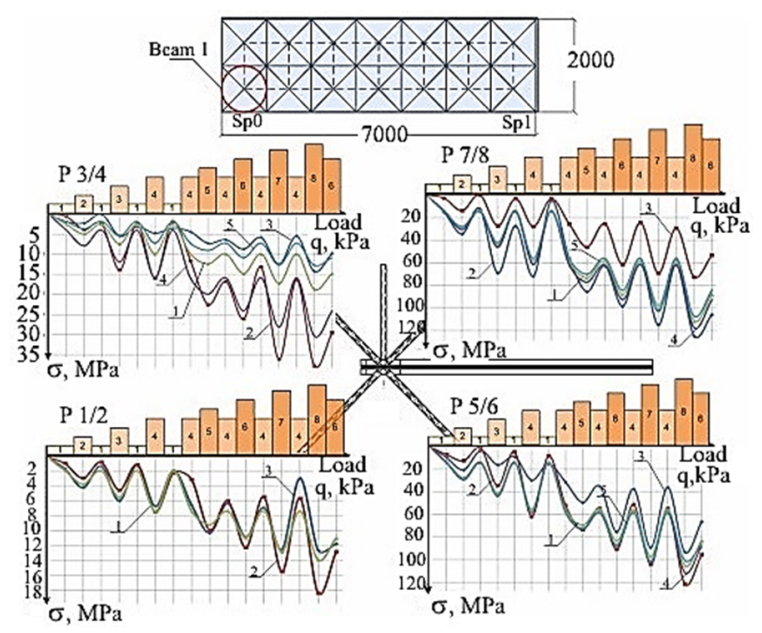

Fig. 8. Stresses in the braces of the superstructure when the entire span is loaded.

As a result of the conducted full-scale tests of the superstructure of the pedestrian bridge, it was established as follows:

- the suggested discrete-continuum system of new type shear connections ensured the joint perfromance of metal structural elements (booms and braces) and a reinforced concrete slab at all loading stages;

- the nature of the system deformation loaded with hydraulic low-cycle short-term and long-term loads corresponds to its resistance data, and the quantitative and qualitative parameters coincide with a sufficient degree of accuracy with the theoretical data of measured values obtained using the constructed finite element model of the design. The discrepancies between the theoretical and experimental data of stresses are within the range of $10-15 \%$;

- vertical displacements under the action of the operational load did not exceed: $f / L=0.0012<0.0025 \cdot L, L-$ span;

- the permanent deformation values after unloading at each loading stage did not exceed $5 \%$, which indicates the elastic performance of the structure;

- no cracks were detected in the reinforced concrete slab, which would characterize the loss of the carrying capacity and the continuity of the structure;

- the period of self-induced vibrations of the structure does not fall within the range prohibited for pedestrian bridges $(0.45$ to $0.6 \mathrm{sec})$;

- reinforcement of the structure by including the reinforced concrete slab in joint performance with the upper boom resulted in an increase of rigidity of the system, on the one hand, and significant increase of its weight, on the other hand. In turn, the increase of the total weight of the structure causes a drop in self-induced vibration frequency. At the same time, it should be noted that an increase in frequency can be achieved by the arrangement of the suggested efficient reinforced concrete slab with internal liners;

- comparison of experimentally obtained dynamic characteristics of the superstructure of the pedestrian bridge with the corresponding theoretical data, as well as in the case of static loading, characterizes the design model as sufficiently correct (the spread of theoretical and experimental data of the studied parameters did not exceed 13-16\%).

The considered designs have been implemented in a number of Ukrainian building companies. The reasons for wide use of this system are as follows:

- a variety of applications (floorings, coverings, bridge superstructures, walls, etc.) (Fig. 9);

- limited height of the structure $(0.04 \div 0.05) L$; 
- the possibility of using it for horizontally, vertically and obliquely installed structures;

- limited consumption of materials;

- low labor intensity of the assembly, which enables to minimize the assembly time.
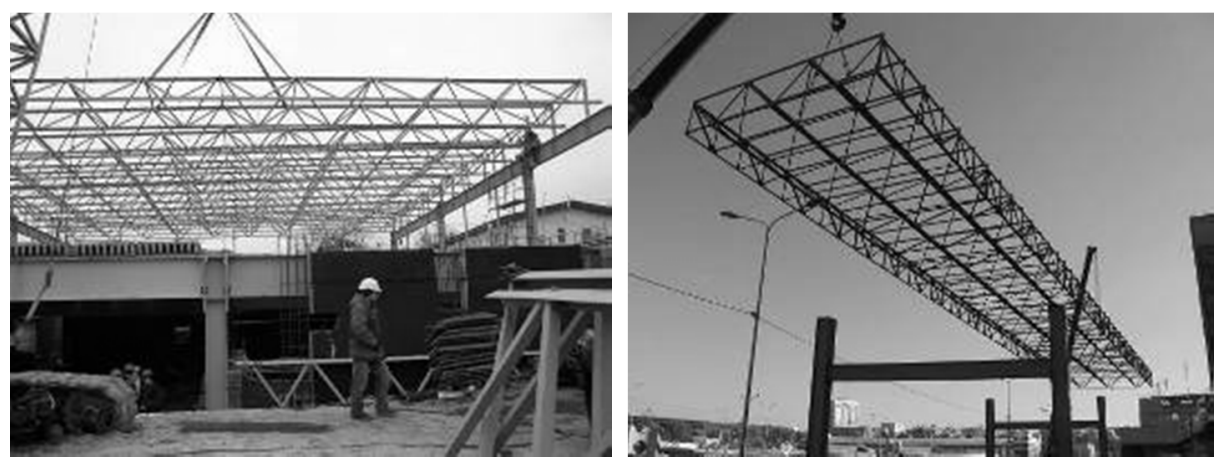

Fig. 9. Facilities where the technology was implemented.

\section{Efficient system of reduce-weight reinforced concrete elements}

\subsection{General}

Reducing the weight of the building is one of the priority issues of the theory of constructions. Minimization of the weight of the structure according to B. Fuller determines the degree of its perfection, comprising such notions as environmental positivity, seismic stability, cost-effectiveness, etc. At the same time, flooring is known to make the principal contribution to the weight of buildings and, in particular, high-rise ones (up to 60 to $70 \%$ ). In this connection, search for rational solutions for these constructions is relevant.

\subsection{Design features}

In $[7,15]$, solutions of monolithic and precast reinforced concrete coverings and floorings, as well as vertical elements, such as walls, pylons, and nuclei, which are structurally anisotropic systems, are suggested. In general, these constructions have the structure of simple external and complex internal geometry (Fig. 10). The main idea here is sinking of liners of agreed shape and sizes made of light, cheap materials such as polystyrene foam, mineral wool, etc. during concreting. Thus the monocoque topology of the constructive element is formed which is similar to bionic systems.

The efficiency of such a design is also determined by the symmetry of its cross-sections which enables its arbitrary support, thus generating a sign-variable field of moments of deflection (Fig. 11).

The above stated is a consequence of the difference in concrete resistance to stretching and compression, on the one hand, and the maximum efficiency (in terms of material consumption) of one-sided ribbed systems, on the other hand. Thus, the construction of the considered slabs consists of two (upper and lower) casings, internal ribs and block-out liners (Fig. 10). Furthermore, if necessary, internal ("hidden") capitals and crossbars are organized inside the element. Reinforcement is a system of flat carcasses located in the ribs (crossbars) and flat grids reinforcing the shells (capitals) (Fig. 11). The outer enclosure of elements, holes and openings are framed with a cut sheet. The installed spacer and internal connection system (usually made of composite materials) provides necessary protective 
layers of concrete and prevents liners from floating. Both light-weight and heavy concrete can be used for constructive elements.

a)

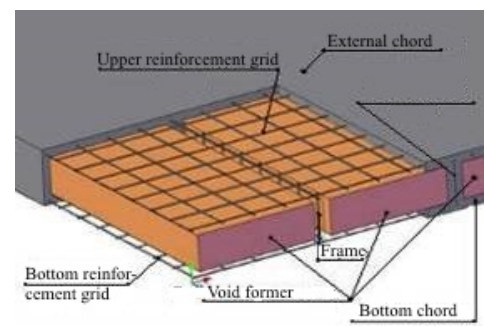

b)

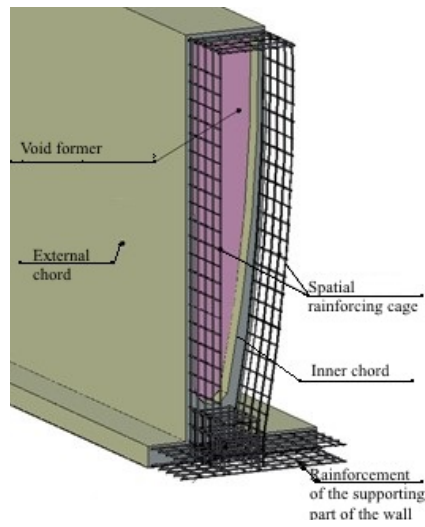

Fig. 10. Topology of the suggested horizontal (a) and vertical (b) efficient elements
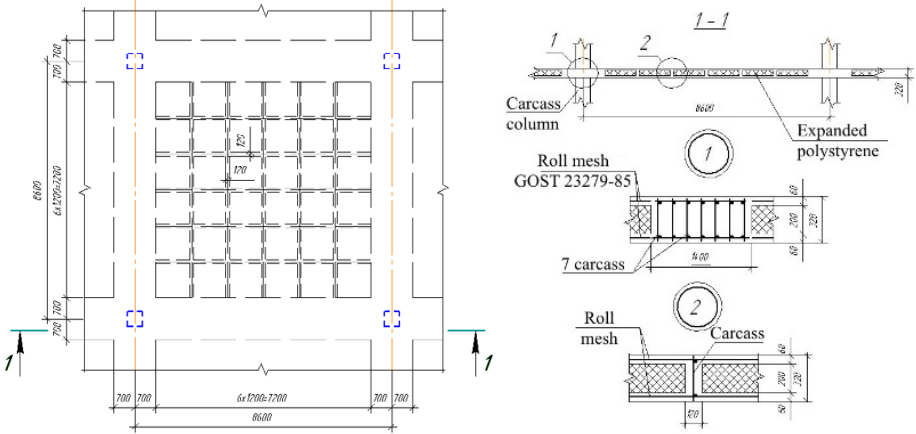

Fig. 11. Fragment of the continuous flooring slab

\subsection{Rationalization of parameters}

The obtained structure with equal structural thicknesses of the solid and the suggested elements enables to save concrete (up to 40-45\%) with the same consumption of reinforcement. Also, the shape and size of the formed internal pockets have significant impact on the nature of the stress-strain state of the system, which explains the expediency of searching for its internal geometry by methods of the control and optimization theory [9, $13,16]$.

In connection with this, we introduce the criterion of rationalization consistent with [9, 13] in the form:

$$
U \rightarrow \inf U\left(\vec{\alpha}^{k}\right), \quad \mathrm{k}=1,2, \ldots \infty,
$$

where $U$ - potential strain energy (PDE); $\mathrm{k}$ - number of comparison variant; $\alpha$-geometric parameter vector; $\alpha \in M$; - set of permissible values of external geometric parameters.

Let us accept requirements of the equal-energy state of the system (construction) as an additional condition to (13), i.e. 


$$
e(\vec{\alpha})=\text { const }
$$

where e - potential strain energy density (PDED).

Solving of practical problems with the criterion (13), to some extent, proves the efficiency of its operation, as well as possibility to regulate not only geometric, but also physical and mechanical characteristics of the system.

Consistently with [9], let us assume that we understand external extensive parameters as parameters which determine resistance of the system without change of the total volume of the material (dimensions of the system elements, bearing connection coordinates and points of load application, etc.), and the internal intensive parameters determine topology, volume, as well as property of the materials.

Equal-energy state caused by (14), as a rational form of existence of artificial elements, ensures the most appropriate distribution of the material, as well as its efficient performance in the construction. If the iterative approach is applied to (14) based on the adaptive evolution method (AEM), for any geometric or physical and mechanical parameter of the construction a formula can be written as follows

$$
h_{i+1}=h_{i}\left[e_{i} e_{i u}^{-1}\right]^{\gamma}
$$

where $h$ - geometric (physical and mechanical) system parameter; $i$ - iteration number; $\gamma \in(0,1)$ - characteristic of adaptive evolution reflecting convergence rate of AEM procedure.

The introduced provisions allow not only to designate a reference solution, but also construct an energy profile of the structure, which is critical. The energy profile of the structure is understood as 3D construction and mapping of the energy density field of deformation with simultaneous determination of the PDE. This fact, along with the definition of the rational system topology (not only form, but also content) predetermines the establishment of the limiting state both for the structural elements of the construction (for example, finite elements) and for the entire system. Moreover, the unloaded (parasitizing) elements of the system are filtered out subject to convergence of procedure (15).

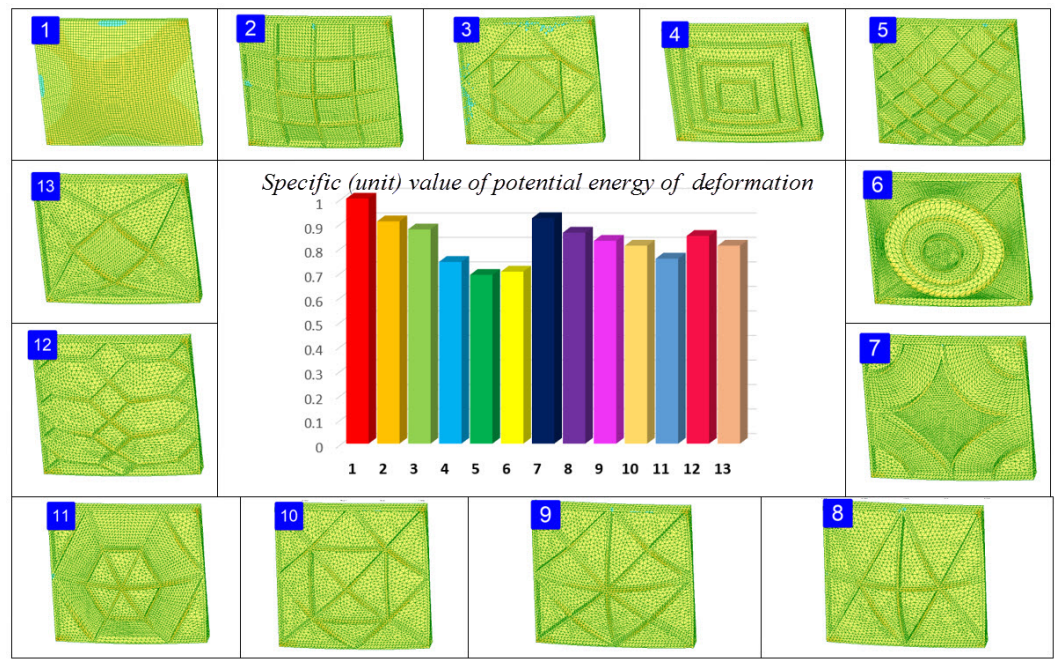

Fig. 12. Illustrated example.

It should be noted that, in addition to minimizing the consumption of materials, the criteria (13) and (14) can be used for approximate estimation of some critical mechanical 
characteristics. In this connection, let us consider an illustrative example. Let us consider 13 square bending constructively anisotropic slabs of the same volume (material consumption). All slabs are supported along the perimeter with hinges and carry uniformly distributed single load. Exclusive geometry of the reinforcing ribs is defined for each slab.

In consistency with the introduced criteria, the task to determine the topology of the edges is set, for which, firstly, the criterion (13) is fulfilled, and secondly, the fulfillment of the limitation (control) is estimated (14). The considered problems were solved in LIRA software environment [10]. The solution results are shown in Figure 12.

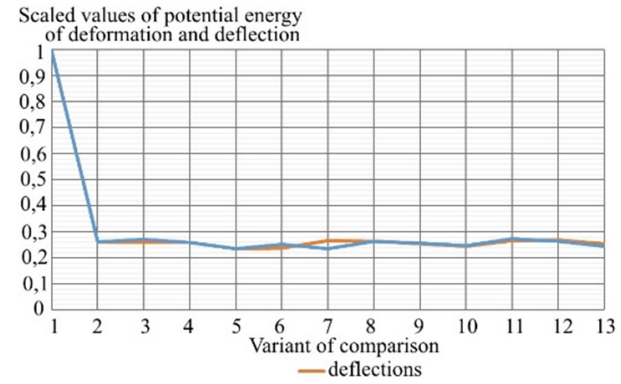

Fig. 13. Maximum deflection and PDE for comparison options.

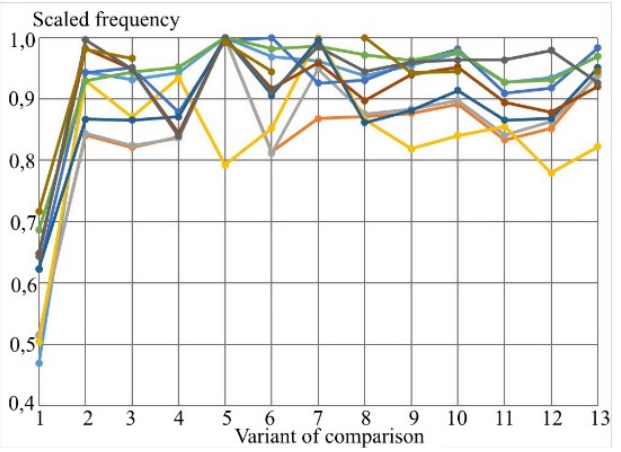

Fig. 14. Self-induced vibration frequencies of the considered slabs.
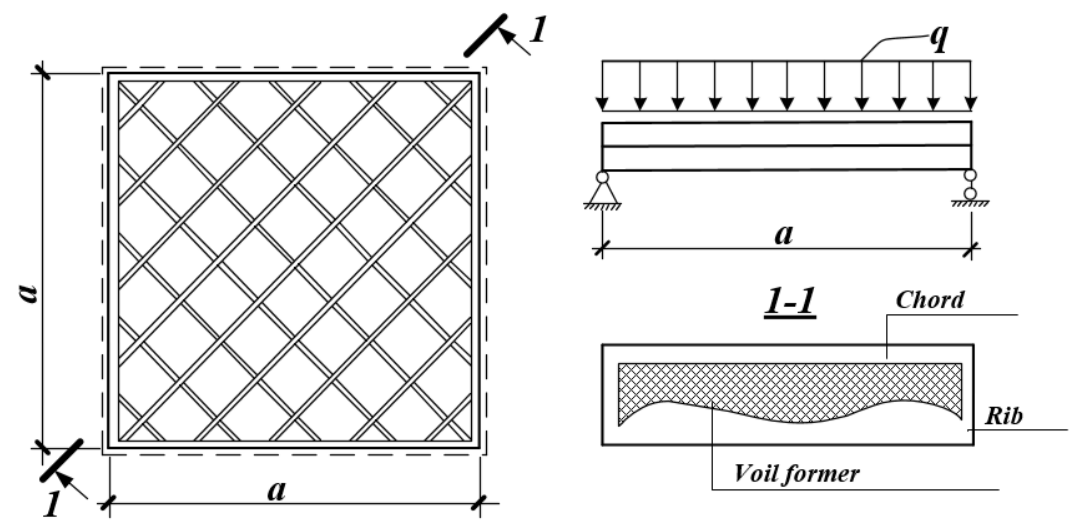

Fig. 15. Ribbed slab with ribs of variable stiffness.

The diagrams in Figures 13, 14 demonstrate, that the fifth option of the slab topology corresponds not only to the minimum value of the PDE, but also to the maximum value of the self-induced vibration frequency of the natural pitch and practically of overtones, as well as the minimum deflection value, which indicates the maximum bending stiffness of 
this slab. Thus (although with a certain degree of caution) we may state that the criterion (13) can be used as a universal one. The condition (14) is fulfilled, inter alia, by variable rigidity of reinforcing ribs $[16,17,18]$. Also, in-depth block-out liners are used to ensure the manufacturability of the solutions (for example, for reinforced concrete or steelreinforced concrete structures) (Fig. 15) [6]. For metal elements, in this case, installation of structural elements with variable cross-section and/or use of metals of different grade can be appropriate [6].

\subsection{Features of the technological process}

Building of the considered systems in monolithic design assumes the use of traditional formwork of the "table" type. The implementation procedure involves concreting in lifts, performed in accordance with the following procedure: installation of the lower mesh and the rib frames (Fig.16a) $\div$ concreting of the bottom shell with concrete compression using the concrete vibrator (Fig.16b) $\div$ laying of the liners and the upper mesh (Fig.16b) $\div$ concreting the ribs and the upper frame (Fig. 16d) with consequent compression with a deep vibrator and beam vibrator $[17,18]$. The work is carried out with pours moving "towards oneself", and the selected mixture composition enables movement on fresh concrete (Fig. 16).
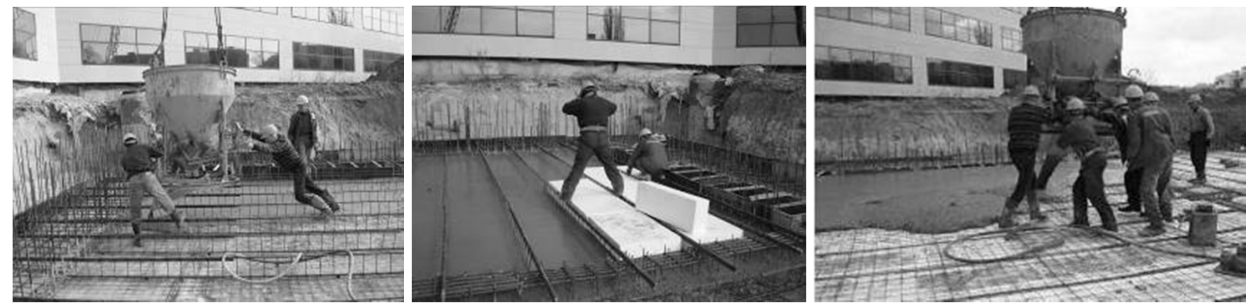

Fig. 16. The building sequence of efficient flooring.

Moulds with opening sides are used in the manufacture of prefabricated elements, while the technological manufacturing process is practically similar to the indicated one (Fig. 17).
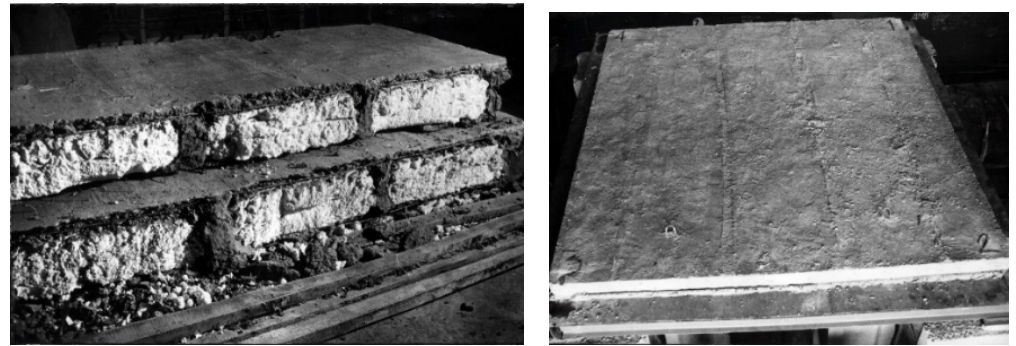

Fig. 17. Efficient prefabricated flooring slab.

There is an advanced alternative technological process using the concrete spraying technology (Fig. 18). It is appropriate to note here that minimization of waste of concrete is ensured with the selected mixture composition and the concreting procedure itself [19]. Nevertheless, off-form concreting is the most important fact. In this case, inserts fixed to the reinforcing special frame play the role of the screen. 

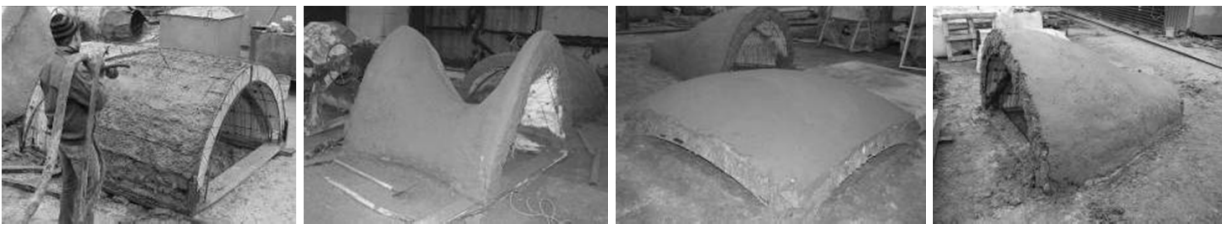

Fig. 18. Off-form concrete spraying (completion).

\subsection{Experimental verification}

Constructive solution, as well as its formation methods were verified experimentally [20]. An object of study was a new type reinforced concrete flooring of monotropic design built above the first floor of a sports and fitness complex under construction. The flooring was a continuous cantilever slab $9.4 \mathrm{~m}$ wide and $43.0 \mathrm{~m}$ long. One side of the slab rested on the caisson supporting wall, and the other rests on the $6 \mathrm{~m}$ span girders that conditionally divide the slab across into the $7.5 \mathrm{~m}$ span and the $1.9 \mathrm{~m}$ console. The structural thickness of the slab is $0.3 \mathrm{~m}$, including the upper and lower plating $0.06 \mathrm{~m}$ and $0.05 \mathrm{~m}$ thick, respectively, and the rest of its height is filled with polystyrene foam liners (Fig. 19). Longitudinal ribs with a width of $0.1 \mathrm{~m}$ are formed inside the plate along the span, located at intervals of $0.75 \mathrm{~m}$. The height of the ribs is equal to the total height of the slab.

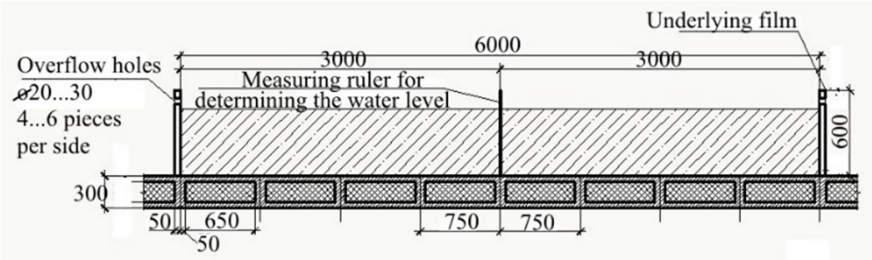

Fig. 19. Flooring cross-section and loading systems.

A new method of full-scale testing of shallow slabs and shells was used to test the efficient flooring $[13,14]$. The loading tanks were assembled from the PERI formwork sections, while waterproofing was ensured with laying three layers of the polyethylene film. The assembled containers were filled with water through the supply pipe to the design level. The overall view of the tank on the flooring is shown in Fig. 20. The water level was regularly measured along the perimeter of the reference enclosure, in the quarters and in the centre of the pool with water to determine the exact nature of loading.
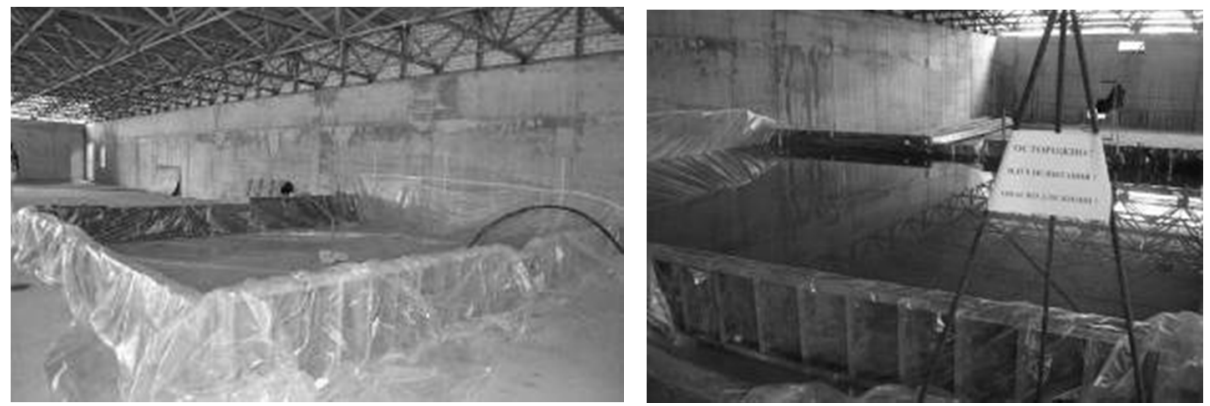

Fig. 20. Overall view of the loading system.

During the experiment, deflections of the characteristics points of the slab were measured using SIIT-3 system. The measurement point arrangement diagram is shown in Fig. 21. 


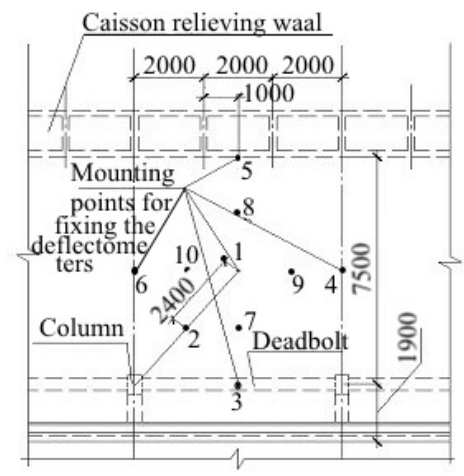

Fig. 21. Sensor arrangement plan.

Loading was performed in incrementing steps of $100 \mathrm{~kg} / \mathrm{m}^{2}$ each $(10 \mathrm{~cm}$ water $)$. Instrument readings were recorded after each supply of the water volume. Then measurements were performed in 10 min (until deformations was stabilized).

The deflection measurement findings at each loading step of the flooring fragment along and across the axes of the tank are given in Figure 22.

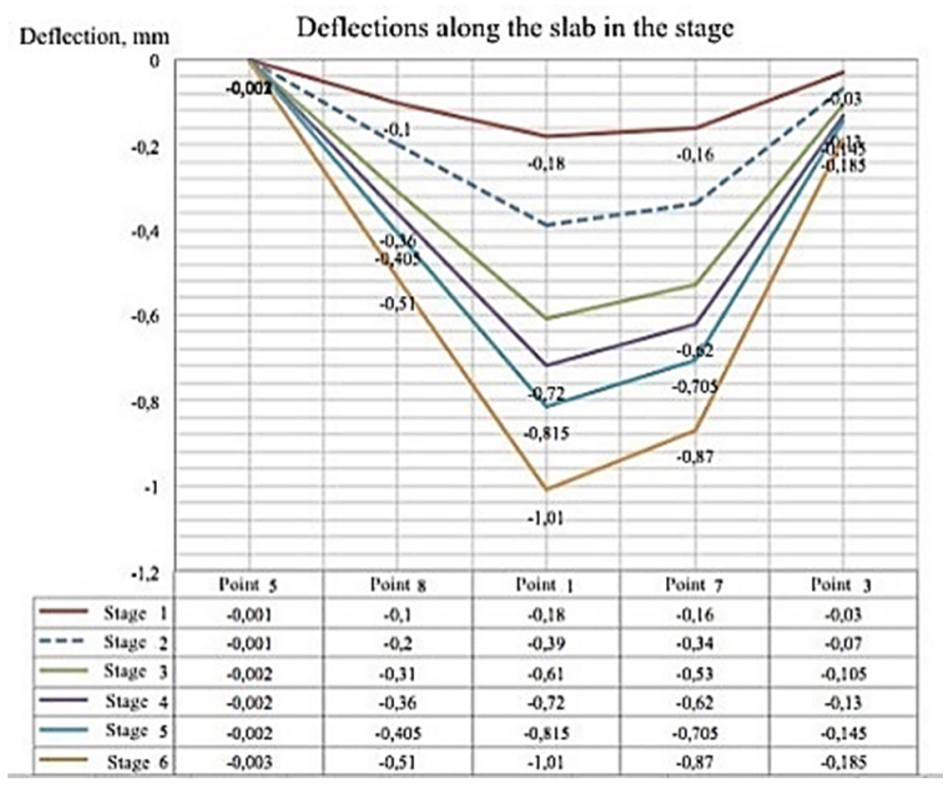

Fig. 22. Deflections of the flooring fragment at loading steps 1-6 along the main instrument arrangement lines: a) deflection lines along the slab span.

Mathematical simulation of the stress-strain state of the research object was performed before testing. Simulation was performed in 3D-finite element form, while the actual calculation was performed in Lira software environment [10]. The analysis of stress-strain state and comparison of theoretical and experimental data confirmed the qualitative and quantitative adequacy of the model (discrepancy within the range of 15\%) (Fig. 23). Moreover, the established nature of the system deformation demonstrated expediency of using the direct design ideology in the suggested form and the above mentioned economic efficiency of the solutions obtained. 

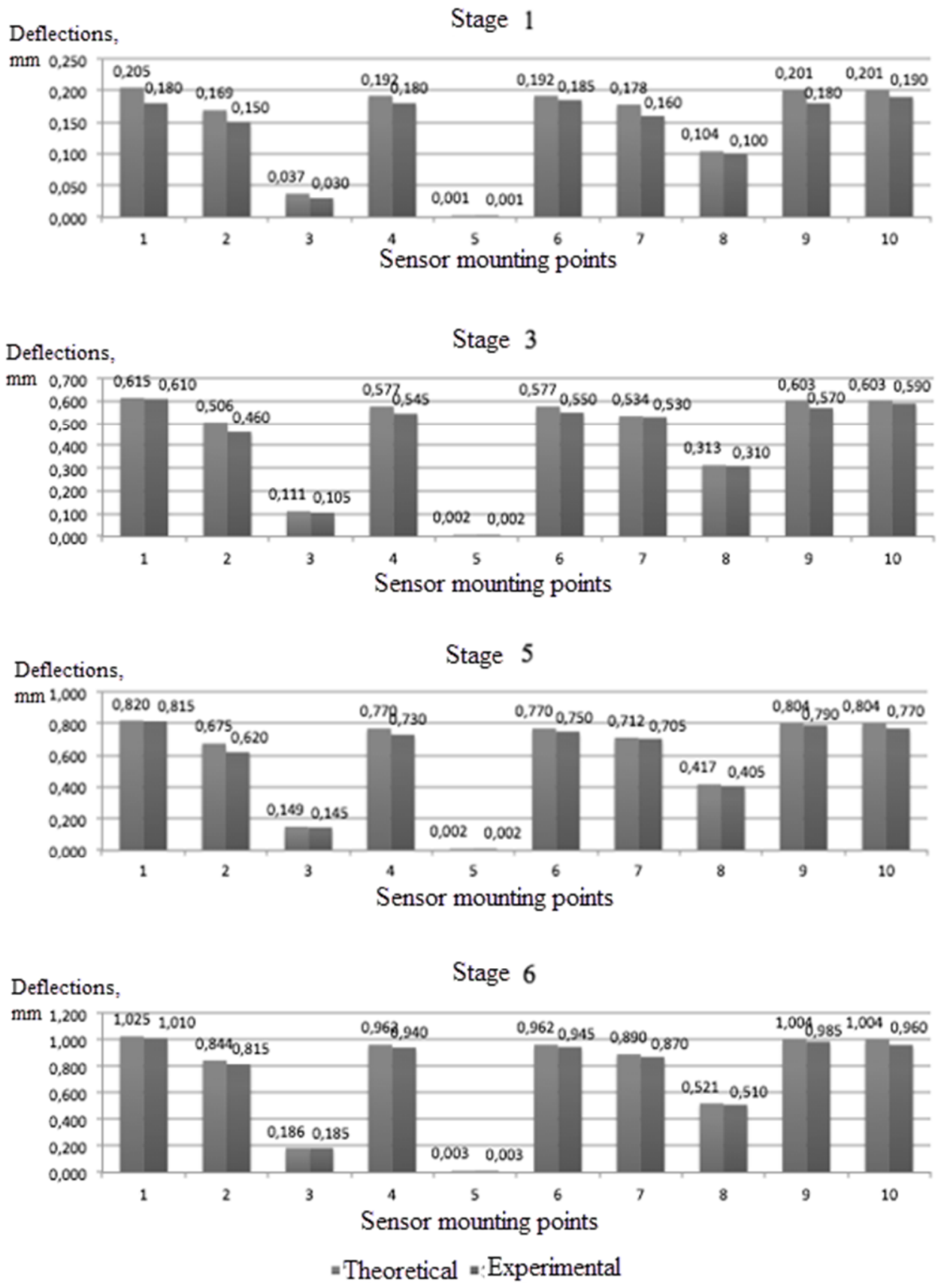

Fig. 23. Comparison graphs for theoretical and experimental deflections in the characteristic points of the slab.

Today, the elaborated designs and technologies have also been introduced and successfully operated in a number of Ukrainian building companies (Fig. 25). 


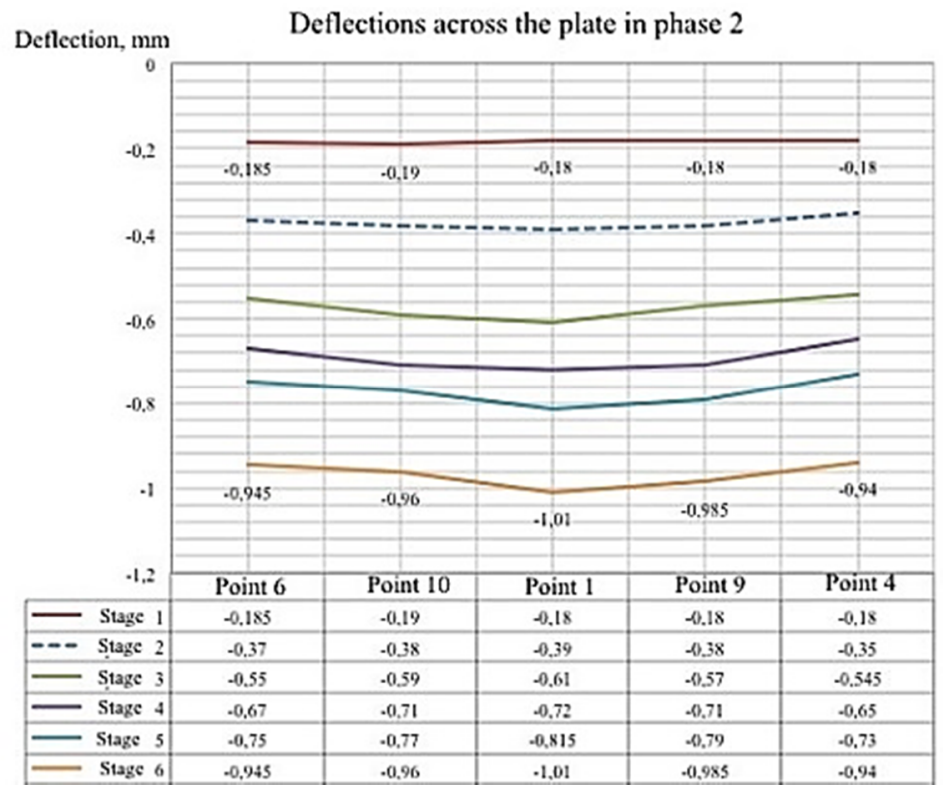

Fig. 24. Deflections of the flooring fragment at loading steps 1-6 along the main instrument arrangement lines: b) deflection lines across the slab span.
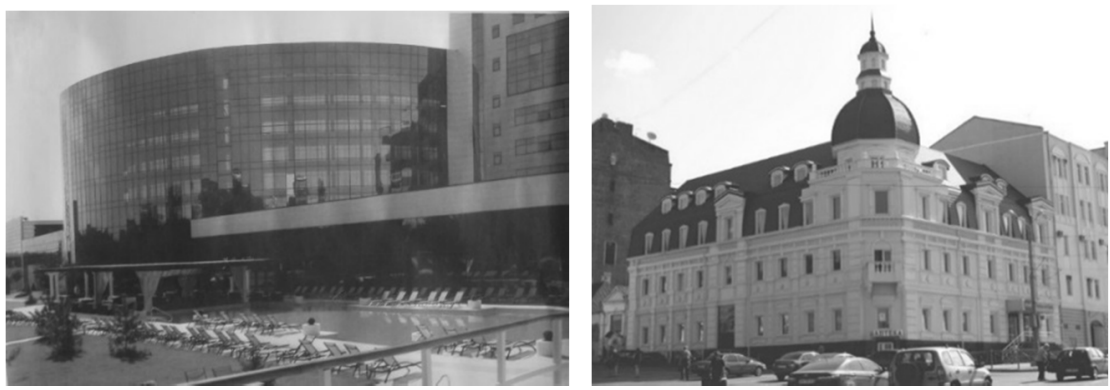

Fig. 25. Facilities containing efficient floor slabs of the suggested design.

In conclusion, it should be noted that the need to save all kinds of resources has always been a dominant factor in the aspiration to improve artefacts. At the same time, the highest integral positivity can be achieved in reasonable transfer to them of solutions borrowed from the wildlife. In turn, the features of the suggested systems and the natural dualism reflected in them are examples of application of concepts given by Galileo:

- if you do not know how to do it - ask Nature;

- no structure can be operated reliably if the form of its ruin (destruction) is unknown.

\section{References}

1. Intelligent buildings: integrated systems and control. Dr Gerard Healey [Electronic resource] // issinstitute.org.au/.../ISS-FEL-REP...-low-res.pdf.

2. EN 1994-1-1-2009 Eurocode 4. Design of building structures, Brussels (2004)

3. V. Shmukler, N. Burak, A. Duker, Vassim Ismail. Patent № 84560, MPK (2006), E04D 3/24, Ukraine (2008).

4. V. Shmukler, E. Beregnaya, V. Gerasimenko, Vassim Ismail, O. Kalmikov. Patent № 51336, MPK (2009), E04D 3/24, Ukraine (2010). 
5. V. Shmukler, A. Fuksman, V. Gerasimenko, Vassim Ismail, O. Kalmikov. Patent № 63846, MPK (2011.01), E04B 1/00, E04D 3/24 (2006/01), Ukraine (2010).

6. V. Shmukler, A. Kislov, E. Krasnova, E.Petrova Proceedings of the $15^{\text {th }}$ Conference for Lithuania Junior Researchers SCIENCE-FUTURE OF LITHUANIA, Vilniuse (2012)

7. V. Shmukler, V. Bedim, I. Shmukler, D. Bedim. Avtorskoe svidetelstvo № 1738962, E04C 2/26, E04B 5/02, USSR (1992).

8. V. Reinboldt, J. Ortega. Iterative methods for solving nonlinear systems of equations Moscow: Mir, 558, (1975).

9. G. Vasilkov. Evolutionary problems of structural mechanics. Synergetic paradigm Rostov-na-Donu: Infoservis (2003).

10. A. Gorodetskiy, V. Shmukler, A. Bondarev. Information technology of calculation and design of building structures. Kharkiv 889 (2003).

11. V. Shmukler. ISEC-03 Third International structural Engineering and construction Conference, Shunan, Japan, 539-545 (2005).

12. V. Shmukler, K. Mohamad. Proceedings of the $2^{\text {th }}$ International Congress FIB, Naples, Italy, 110-127 (2006).

13. V. Shmukler, U. Klimov, N. Burak. Light-weight frame systems, Kharkov, 336, (2008).

14. V. Shmukler, A. Chuprynin, R. Abbasi Patent № 44125. MPK (2009), G01M 19.00, G01N 3.00, G01M 5.00, Ukraine (2009).

15. V. Shmukler, O. Kalmikov, E. Lugchenko, A. Goishin, V. Zinchenko. Patent № 79485, MPK, E02D 29/02 (2006.01), Ukraine (2013).

16. V. Shmukler. Global Construction, Dundee, Scotland, 183-190 (2005).

17. V. Shmukler, M. Pomazan. Patent №69346, MPK (2012.01), E04C 2/00, E04B 5/00, E04G 21/00, Ukraine (2012).

18. V. Shmukler, M. Pomazan. Patent №75556, MPK (2012.01), E04C 2/00, E04B 5/00, E04G 21/00, Ukraine (2012).

19. S. Bugaevskiy. «Naukovo-technichni problems suchasnogo zalizobetonu» 78 (2), 478-586 (2013).

20. I. Steblovskii, E. Beregnaya Zbirnyk naukovych pratc «Budivelni konstruktsii», 79, 347-355 (2013). 